\title{
Tecnura
}

\section{Evaluación y comparación del análisis granulométrico obtenido de agregados naturales y reciclados}

\author{
Evaluation and comparison of the grain size analysis \\ obtained from natural and recycled aggregates
}

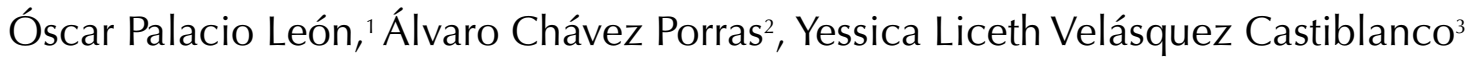

Fecha de recepción: 2 de noviembre de 2015

Fecha de aceptación: 18 de noviembre de 2016

Cómo citar: Palacio-Leon, O., Chávez, A., y Velásquez, Y. (2017). Evaluación y comparación del análisis granulométrico obtenido de agregados naturales y reciclados. Revista Tecnura, 21(53), 96-106, doi: 10.14483/22487638.8195

\section{Resumen}

Contexto: el uso de residuos de construcción y demolición (RCD) hace parte de la gestión que permite mitigar contaminación ambiental y disminuye el impacto que ocasiona la extracción de agregados naturales. La industria del sector de la construcción posee en marcha objetivos de implementación de la metodología de las 3R (reducir, reusar y reciclar) y los procesos de producción más limpia para los RCD; siendo en Colombia un sector con crecimiento anual del 13\%, lo que incrementa la disposición.

Método: se requirió establecer un análisis granulométrico comparativo entre los agregados naturales y reciclados, que permitió identificar desde el punto de vista técnico, semejanzas y diferencias, entre parámetros establecidos en la Norma Técnica Colombiana NTC 174 (especificaciones de agregados en la producción de concretos). Las muestras de agregados reciclados fueron tomadas de escombreras artesanales de la ciudad de Bogotá D.C., y las muestras de agregados naturales obtenidas del sector comercial, en la ciudad.
Resultados: se obtuvieron curvas granulométricas de los agregados respecto a los límites establecidos por la norma NTC 176, lográndose determinar que los RCD no cumplen con las especificaciones normativas de los concretos; sin embargo, no se descarta su uso como agregado para el concreto no estructural, para la fabricación modular sostenible.

Conclusiones: se requiere de una evaluación y comparación granulométrica de los agregados para establecer que los residuales sean reutilizados como materia prima, promoviendo una política ambiental que sugiera el uso como estrategia de sostenibilidad en entornos sociales.

Palabras clave: agregado natural, agregado reciclado, análisis granulométrico, RCD, residuos de construcción y demolición.

\begin{abstract}
Context: The use of construction and demolition waste-CDW, is part of the management that allows us to mitigate environmental pollution and reduces the impact caused by the extraction of natural aggregates. The sector of construction industry has launched aims
\end{abstract}

1 Ingeniero Industrial, especialista en Evaluación y Desarrollo de Proyectos, magister en Ingeniería Industrial y magister en Investigación Operativa y Estadística. Docente de la Universidad Militar Nueva Granada. Bogotá D.C., Colombia. Contacto: oscar.palacio@unimilitar.edu.co

2 Ingeniero Industrial, doctor en Ingeniería Civil Área Ambiental. Docente de la Universidad Militar Nueva Granada. Bogotá D.C., Colombia. Contacto: alvaro.chavez@unimilitar.edu.co

3 Ingeniera Industrial, especialista en Gerencia en Logística Integral. Docente de la Universidad Militar Nueva Granada. Bogotá D.C., Colombia. Contacto: yessica.velasquez@unimilitar.edu.co 
of implementation of "3R-reduce, reuse and recycle" methodology and "Cleaner production" for the CDW processes; being in Colombia, a sector with annual growth of $13 \%$, which increases the available.

Method: It was required to establish a comparative particle size analysis between aggregate natural and recycled, which allowed to identify from the technical point of view, similarities and differences between parameters established in the Colombian technique standard NTC 174 (specifications for aggregates in concrete production). Samples of recycled aggregates were taken from artisanal dumps of Bogotá D.C.; and samples of natural aggregates, obtained from the commercial sector in the city.
Results: We obtained grain size curves of the aggregates regarding the limits set by the standard NTC 176. Achieving determines that the CDW do not comply with the normative specifications of the concrete. However it is possible to use as aggregate for concrete structural, no sustainable modular manufacturing.

Conclusions: It is needed an assessment and grading comparison of the aggregates to establish that the residuals are reused as raw material: promoting an environmental policy that suggests use as a sustainability strategy in social settings.

Keywords: construction and demolition waste, grain size analysis, natural aggregate, recycled aggregate.

\section{INTRODUCCIÓN}

Con la continua explotación de agregados naturales del subsuelo, surge la necesidad de hacer un continuo análisis del impacto ambiental y económico que esta actividad genera para el ecosistema; situación a la que aúna la ausencia de regulaciones de control sobre las explotaciones de los mismos y sus respectivas limitaciones (Scanferla y Barreda, 2011; Wu, Yu, Shen, y Liu, 2014).

Para compensar este desgaste natural han surgido diferentes metodologías, principalmente las relacionadas al reúso y reciclaje de materiales, como es el caso de Alemania, considerada de las primeras naciones en usar agregados reciclados en el sector de la construcción, dada la gran generación de residuos de construcción y demolición (RCD), producto de la Segunda Guerra Mundial, fenómeno acaecido como consecuencia de los enfrentamientos bélicos acontecidos en su territorio. Pero en cuanto a investigaciones adelantadas en este campo, se cree que tuvieron lugar por primera vez en Rusia en 1946; desde entonces, la necesidad de crear entornos y ejes de acción que permitan realizar una gestión adecuada de los RCD, ha sido el objetivo de los estudios de gran parte de los países desarrollados del mundo, siendo Europa el continente con mayor nivel de investigación y aplicación, donde España, por ejemplo, ha reglamentado el uso de agregados reciclados en concretos estructurales y no estructurales, a través de la norma "Instrucción de Concreto Estructural EHE-08" (Kumutha y Vijai, 2010; Viudaud, Castaño , y Vidaud, 2013; Cheng y Ma, 2013).

A nivel global, el uso de agregados reciclados es una práctica relevante que tiene como propósito prevenir la contaminación ambiental y disminuir el impacto que ocasiona la extracción de agregados naturales. Grandes compañías dedicadas a la industria de la construcción, o multinacionales productoras de materiales para esta, esperan que a corto plazo los residuos de materiales empleados en cimentaciones, sean materia prima de en este tipo de producción; considerando que los agregados conforman al menos el $75 \%$ de una mezcla de concreto convencional, entendiéndose como agregado la mezcla entre arena, grava natural y piedra triturada (Méndez, Rodríguez, Cruz, y Laines, 2010; Ulsen, et al., 2013).

En Colombia, la extracción intensiva de materias primas naturales para la industria de la construcción ha aumentado en proporción al incremento presentado por dicho sector, el cual representa una tasa de crecimiento del $13,2 \%$ anual; 
en consecuencia, se presenta un aumento en la generación de RCD y se crea la necesidad de gestionar sus procesos de disposición y reciclaje. En Bogotá D.C., se estima que la producción de RCD para 2013 alcanzó los 13000000 m³ (Vega, 2013).

A pesar de las ventajas que genera su uso y las investigaciones adelantadas en el campo, gran parte de ellos no son empleados como insumos sustentables en las obras civiles actuales (Castaño, Misle, Lasso, Gómez, y Ocampo, 2013; Wang, Wu, y Zhang, 2013).

Los análisis comparativos de agregados naturales y reciclados permiten, desde el punto de vista técnico, observar las semejanzas y diferencias que se presentan, a nivel físico, siendo el análisis granulométrico el criterio de selección del material reciclado, en dosificación determinada, con la normativa NTC 174 "Concretos. Especificaciones de los agregados para concreto" (Carrasco, 2014), donde los agregados reciclados no cumplieron con las características físicas requeridas para ser empleados totalmente como elementos en las obras civiles. Permitiendo su uso solo con mezclas previamente estudiadas, perseverando en su eje de acción desde el ámbito industrial y ambiental, ya que se garantiza la reducción del riesgo de agotamiento de los naturales, así como el aumento de la reutilización adecuada de RCD. Obteniendo "valor agregado", basados en las nuevas tecnologías de sostenibilidad y promoción de la reducción del impacto ambiental que generan los RCD, a través de su reciclaje.

\section{MARCO TEÓRICO}

Los agregados, son materiales inertes con forma granular, considerados como productos minerales que pueden encontrarse en estado natural o artificial, y cuya mezcla con un cementante permite generar concreto. De acuerdo a su procedencia, pueden clasificarse en naturales y artificiales (Yuan, Shen, y Li , 2011).

Los agregados naturales son aquellos que provienen de la explotación de fuentes naturales y cuyas partículas se generan como consecuencia de procesos naturales como intemperismo o abrasión; por otro lado, los agregados artificiales se producen a partir de técnicas industriales especializados en el proceso de trituración de los materiales de los cuales proceden (Rivera, 2013; Banias, AchiIlas, Vlachokostas, Moussiopoulos, y Papaioannou, 2011).

De acuerdo a su tamaño, los agregados se clasifican en agregados finos y gruesos; los agregados finos, conocidos como arena, son aquellos que pasan a través del tamiz número cuatro, equivalente a 4,76 mm. Por su parte, los agregados gruesos denominados en la industria como grava, superan en tamaño el diámetro fijado en el tamiz número cuatro (Méndez, Rodríguez Jiménez, Cruz, y Laines, 2011).

Por su parte, los agregados reciclados, son aqueIlos que provienen de la recuperación y el tratamiento de los RCD; son residuos inertes generados en obras de construcción, reparación, remodelación y demolición de edificios e infraestructuras (Srour, Chehab, El-Fadel, y Tamraz, 2013). Se considera que conforman el $75 \%$ de los residuos generados en la obra, el 25\% restante generalmente son clasificados como tóxicos o peligrosos (De Santos, Monercillo, y García, 2011; Leiva , Solís, Marrero, y García, 2013).

Esta clasificación, se realiza a través del análisis granulométrico, el cual hace referencia a la distribución del tamaño de las partículas de un agregado; dicho análisis se determina a través del paso de una muestra representativa de agregado por una serie de tamices, cuyas aberturas se ordenan de mayor a menor. Entendiéndose por tamiz una malla de abertura cuadrada debidamente estandarizado, de acuerdo a las especificaciones dadas en la tabla 1; es necesario resaltar, que la gradación genera un acomodamiento de las partículas y aumenta el nivel de resistencia a la flexión y compresión (Paredes, Reyes, y Camacho, 2014; Molina, Camacho, y Reyes, 2016).

El estudio de los resultados se da de acuerdo al porcentaje de agregado retenido $y$, en 
consecuencia, al porcentaje del mismo que pasa a través del tamiz; de esta manera, se establece un control gráfico para los análisis conocidos como curvas granulométricas, caracterizadas por representar en el eje de las abscisas la abertura del tamiz y en el eje de las ordenadas el porcentaje de agregado que pasa por el tamiz respectivo, las cuales se construyen a partir del uso de los modelos establecidos en las ecuaciones (1) y (2).

$$
\begin{gathered}
\% \text { Retenido }=\frac{\text { Peso de material retenido en tamiz }}{\text { Peso total de la muestra }} * 100 \\
\% \text { Pasa }=100-\% \text { Retenido acumulado }
\end{gathered}
$$

Tabla 1. Tamaños y designación de tamices ASTM

\begin{tabular}{ccccc}
\hline Posición & Abertura (pulg.) & Abertura $(\mathbf{m m})$ & Designación tamiz & Módulo de finura \\
\hline 0 & $3 / 1024$ & $0,0744 \sim 0,075$ & $\# 200$ & 0 \\
\hline 1 & $3 / 512$ & $0,149 \sim 0,150$ & $\# 100$ & 1 \\
\hline 2 & $3 / 256$ & $0,298 \sim 0,300$ & $\# 50$ & 2 \\
\hline 3 & $3 / 128$ & $0,595 \sim 0,600$ & $\# 30$ & 3 \\
\hline 4 & $3 / 64$ & $0,196 \sim 1,20$ & $\# 16$ & 4 \\
\hline 5 & $3 / 32$ & $2,381 \sim 2,40$ & $\# 8$ \\
\hline 6 & $3 / 16$ & $4,762 \sim 4,80$ & $\# 4$ & 6 \\
\hline 7 & $3 / 8$ & $9,52 \sim 9,5$ & $3 / 8^{\prime \prime}$ & 7 \\
\hline 8 & $3 / 4$ & $19,05 \sim 19$ & $3 / 4^{\prime \prime}$ & 8 \\
\hline 9 & $3 / 2$ & $38,1 \sim 38$ & $1.5^{\prime \prime}$ & 9 \\
\hline 10 & 3 & $76,2 \sim 76$ & $3{ }^{\prime \prime}$ & 10 \\
\hline
\end{tabular}

Fuente: (Giraldo, 2003)

Es necesario resaltar, que el análisis granulométrico permite establecer el valor de los siguientes criterios:

Tamaño máximo: Donde se hace referencia a la dimensión de la mayor partícula que se encuentra inmersa en la muestra. Es la menor abertura de un tamiz respectivo, por la cual pasa la totalidad de la muestra a analizar.

Tamaño máximo nominal: Donde se hace referencia a la dimensión del tamiz superior al cual el porcentaje retenido acumulado es superior o igual al 15\%.

Módulo de finura (Mf): Donde se permite identificar el grosor de las partículas de la muestra analizada, se establece de acuerdo al modelo presentado a través de la ecuación (3).

$$
\mathrm{Mf}=\frac{\sum \% \text { Retenido Acumulado }}{100}
$$

Porcentaje de finos: Es el valor porcentual de la muestra que pasa el tamiz $\mathrm{N}^{\circ} 200$ o de $75 \mu \mathrm{m}$.

El concreto es una mezcla de un material aglutinante, generalmente cemento, material de relleno, como agregados finos y gruesos, agua y si es requerido aditivos; actualmente, es considerado como uno de los materiales de mayor uso en la industria de la construcción, gracias a la gran variedad de elementos estructurales y no estructurales que pueden fabricarse a partir este (Sánchez, 2001; Ding y Xiao, 2014). 
La mezcla de concreto se encuentra establecida en la proporción 1-2-4, en relación a cemento-arena-grava respectivamente, lo cual indica que debe estar compuesto por una parte de cemento (14\%), dos partes de arena (29\%) y cuatro partes de grava (57\%); estas proporciones, deben encontrarse relacionadas al peso, actualmente las dosificaciones respecto al volumen son menos empleadas (JaramiIlo, 2014).

De acuerdo a las especificaciones establecidas en la NTC 174 "Concretos. Especificaciones de los agregados para concreto", el agregado fino que ha de ser empleado para concreto, debe estar compuesto de arena natural, arena triturada o una combinación de estos. Adicionalmente, se establece que ha de cumplir con los umbrales indicados en la tabla 2 y el módulo de finura debe encontrarse entre 2,3 y 3,1 .

Por su parte, el agregado grueso debe estar compuesto de grava, grava triturada, roca triturada,
Tabla 2. Especificaciones de gradación del agregado fino empleado para concreto

\begin{tabular}{cc}
\hline Tamiz NTC 32 (ASTM E 11) & Porcentaje que pasa \\
\hline $9,5 \mathrm{~mm}$ & 100 \\
\hline $4,75 \mathrm{~mm}$ & 95 a 100 \\
\hline $2,35 \mathrm{~mm}$ & 80 a 100 \\
\hline $1,18 \mathrm{~mm}$ & 50 a 85 \\
\hline $600 \mu \mathrm{m}$ & 25 a 60 \\
\hline $300 \mu \mathrm{m}$ & 10 a 30 \\
\hline $150 \mu \mathrm{m}$ & 2 a 10 \\
\hline
\end{tabular}

Fuente: Instituto Colombiano de Normas Técnicas y Certificación (ICONTEC, 2000).

escoria de alto horno enfriada al aire (desecho ácido del material de hierro y cenizas de azufre del coque), concreto fabricado con cemento hidráulico o con una combinación de ellos. De igual forma, debe cumplir con las parametrizaciones fijadas en la tabla 3.

Tabla 3. Especificaciones de gradación del agregado grueso empleado para concreto

\begin{tabular}{|c|c|c|c|c|c|c|c|c|c|c|c|c|c|c|}
\hline \multirow{2}{*}{$\begin{array}{c}\text { Número } \\
\text { del } \\
\text { tamaño } \\
\text { del } \\
\text { agregado }\end{array}$} & \multirow{2}{*}{$\begin{array}{c}\text { Tamaño nominal } \\
\text { (tamices de abertura } \\
\text { cuadrada) }\end{array}$} & \multicolumn{13}{|c|}{ Material que pasa uno de los siguientes tamices (porcentaje en masa) } \\
\hline & & $\begin{array}{l}100 \\
\mathrm{~mm}\end{array}$ & 90 mm & $\begin{array}{c}75 \\
\mathrm{~mm}\end{array}$ & $63 \mathrm{~mm}$ & $50 \mathrm{~mm}$ & $\begin{array}{l}37,5 \\
\mathrm{~mm}\end{array}$ & $\begin{array}{l}25,0 \\
\mathrm{~mm}\end{array}$ & $\begin{array}{l}19,0 \\
\mathrm{~mm}\end{array}$ & $\begin{array}{l}12,5 \\
\mathrm{~mm}\end{array}$ & $\begin{array}{l}9,5 \\
\mathrm{~mm}\end{array}$ & $\begin{array}{c}4,75 \mathrm{~mm} \\
\left(\mathrm{~N}^{\circ} 4\right)\end{array}$ & $\begin{array}{c}2,36 \mathrm{~mm} \\
\left(\mathrm{~N}^{\circ} 8\right)\end{array}$ & $\begin{array}{r}1,18 \mathrm{~mm} \\
\left(\mathrm{~N}^{\circ} 16\right)\end{array}$ \\
\hline 1 & $90 \mathrm{~mm}$ a $37,5 \mathrm{~mm}$ & 100 & $90-100$ & - & $25-60$ & - & $0-15$ & - & $0-5$ & - & - & - & - & - \\
\hline 2 & $63 \mathrm{~mm}$ a $37,5 \mathrm{~mm}$ & - & - & 100 & $90-100$ & $35-70$ & $0-15$ & - & $0-5$ & - & - & - & - & - \\
\hline 3 & $50 \mathrm{~mm}$ a $25,0 \mathrm{~mm}$ & - & - & - & 100 & $90-100$ & $35-70$ & $0-15$ & - & $0-5$ & - & - & - & - \\
\hline 357 & $50 \mathrm{~mm}$ a $4,75 \mathrm{~mm}\left(\mathrm{~N}^{\circ} 4\right)$ & - & - & - & 100 & 95-100 & - & $35-70$ & - & $10-30$ & - & $0-5$ & - & - \\
\hline 4 & $37,5 \mathrm{~mm}$ a $19,00 \mathrm{~mm}$ & - & - & - & - & 100 & $90-100$ & $20-55$ & $0-15$ & - & $0-5$ & - & - & - \\
\hline 467 & $\begin{array}{l}37,5 \mathrm{~mm} \text { a } 4,75 \mathrm{~mm} \\
\left(\mathrm{~N}^{\circ} 4\right)\end{array}$ & - & - & - & - & 100 & $95-100$ & - & $35-70$ & - & $\begin{array}{c}10- \\
30\end{array}$ & $0-5$ & - & - \\
\hline 5 & $25,0 \mathrm{~mm}$ a $12,5 \mathrm{~mm}$ & - & - & - & - & - & 100 & $90-100$ & $20-55$ & $0-10$ & $0-5$ & - & - & - \\
\hline 56 & $25,0 \mathrm{~mm}$ a $9,5 \mathrm{~mm}$ & - & - & - & - & - & 100 & $90-100$ & $40-85$ & $10-40$ & $0-15$ & $0-5$ & - & - \\
\hline 57 & $\begin{array}{l}25,0 \mathrm{~mm} \text { a } 4,75 \mathrm{~mm} \\
\left(\mathrm{~N}^{\circ} 4\right)\end{array}$ & - & - & - & - & - & 100 & $95-100$ & - & $25-60$ & - & $0-10$ & $0-5$ & - \\
\hline 6 & $19,0 \mathrm{~mm}$ a $9,5 \mathrm{~mm}$ & - & - & - & - & - & - & 100 & $90-100$ & $20-55$ & $0-15$ & $0-5$ & - & - \\
\hline 67 & $\begin{array}{l}19,0 \mathrm{~mm} \text { a } 4,75 \mathrm{~mm} \\
\left(\mathrm{~N}^{\circ} 4\right)\end{array}$ & - & - & - & - & - & - & 100 & $90-100$ & - & $\begin{array}{c}20- \\
55\end{array}$ & $0-10$ & $0-5$ & - \\
\hline 7 & $\begin{array}{l}12,5 \mathrm{~mm} \text { a } 4,75 \mathrm{~mm} \\
\left(\mathrm{~N}^{\circ} 4\right)\end{array}$ & - & - & - & - & - & - & - & 100 & $\begin{array}{c}90- \\
100 \\
\end{array}$ & $\begin{array}{l}40- \\
70\end{array}$ & $0-15$ & $0-5$ & - \\
\hline 8 & $\begin{array}{l}9,5 \mathrm{~mm} \text { a } 2,36 \mathrm{~mm} \\
\left(\mathrm{~N}^{\circ} 8\right)\end{array}$ & - & - & - & - & - & - & - & - & 100 & $\begin{array}{l}85- \\
100\end{array}$ & $10-30$ & $0-10$ & $0-5$ \\
\hline
\end{tabular}

Fuente: Instituto Colombiano de Normas Técnicas y Certificación (ICONTEC, 2000) 


\section{METODOLOGÍA}

El protocolo que siguió el proceso de experimentación, se puede resumir en los siguientes pasos que están soportados por el método científico.

Las muestras de agregados reciclados, utilizados en el estudio, fueron tomadas de una escombrera artesanal de la ciudad de Bogotá D.C., ubicada en la Localidad Ciudad Bolívar, en la cual se lleva a cabo el proceso de trituración de concreto y clasificación del agregado por tamaño de partícula. Adicionalmente, las muestras de agregados naturales fueron obtenidas del sector comercial, en la ciudad.

- Los análisis granulométricos fueron Ilevados a cabo en laboratorios de suelos apropiados y debidamente homologados por el ente regulador en medio ambiente de Colombia, observando:

- Peso retenido.

- Porcentaje retenido.

- Porcentaje retenido acumulado.

- Porcentaje que pasa cada uno de los tamices.
- Se desarrolló un proceso de mezclado con la siguiente participación de material: por tres partes de muestras de agregados reciclados (M1, M2 y M3) se adiciono una de agregados naturales $(N)$, la cual busca la obtención de curvas granulométricas de los agregados respecto a los límites establecidos por la norma NTC 176.

- Finalizando con la comparación de los resultados obtenidos para los diferentes grupos de muestras, con respecto a las especificaciones técnicas expuestas en la norma NTC 174 "Concretos. Especificaciones de los agregados para concreto".

\section{RESULTADOS}

El análisis granulométrico realizado a las muestras del estudio, permitió observar el peso retenido, porcentaje retenido, porcentaje retenido acumulado y porcentaje que pasa cada uno de los tamices empleados en el mismo; lo anterior, puede evidenciarse en la tabla 4 y la tabla 5 para agregados reciclados y naturales respectivamente. Adicionalmente, el comportamiento de los datos, se mostró en la figura 1 y la figura 2 .

Tabla 4. Análisis granulométrico agregados reciclados

\begin{tabular}{cccccc}
\hline Tamiz $\mathbf{N}^{\circ}$ & Diámetro $(\mathbf{m m})$ & Peso Retenido $(\mathbf{g})$ & \% Retenido & \% Retenido Acumulado & \% Pasa \\
\hline $2^{\prime \prime}$ & 50,800 & 0,00 & 0,00 & 0,00 & 100,00 \\
\hline $11 / 2^{\prime \prime}$ & 36,100 & 0,00 & 0,00 & 0,00 & 100,00 \\
\hline $1^{\prime \prime}$ & 25,400 & 0,00 & 0,00 & 0,00 & 100,00 \\
\hline $3 / 4^{\prime \prime}$ & 19,050 & 11,72 & 1,09 & 1,09 & 98,91 \\
\hline $1 / 2^{\prime \prime}$ & 12,700 & 18,73 & 1,75 & 2,84 & 97,16 \\
\hline $3 / 8^{\prime \prime}$ & 9,520 & 32,75 & 3,05 & 5,89 & 94,11 \\
\hline 4 & 4,750 & 174,33 & 16,25 & 22,15 & 77,85 \\
\hline 10 & 2,000 & 285,92 & 26,66 & 48,81 & 51,19 \\
\hline 30 & 0,600 & 237,23 & 22,12 & 70,93 & 29,07 \\
\hline 40 & 0,425 & 65,28 & 6,09 & 77,01 & 22,99 \\
\hline 60 & 0,250 & 117,79 & 10,98 & 87,99 & 12,01 \\
\hline 100 & 0,150 & 83,95 & 7,83 & 95,82 & 4,18 \\
\hline 200 & 0,074 & 42,15 & 3,93 & 99,75 & 0,25 \\
\hline Fondo & & 2,42 & 0,23 & 99,98 & 0,02 \\
\hline Total error (\%) & 0,022 & 1072,27 & 99,98 & - & - \\
\hline
\end{tabular}

Fuente: elaboración propia 


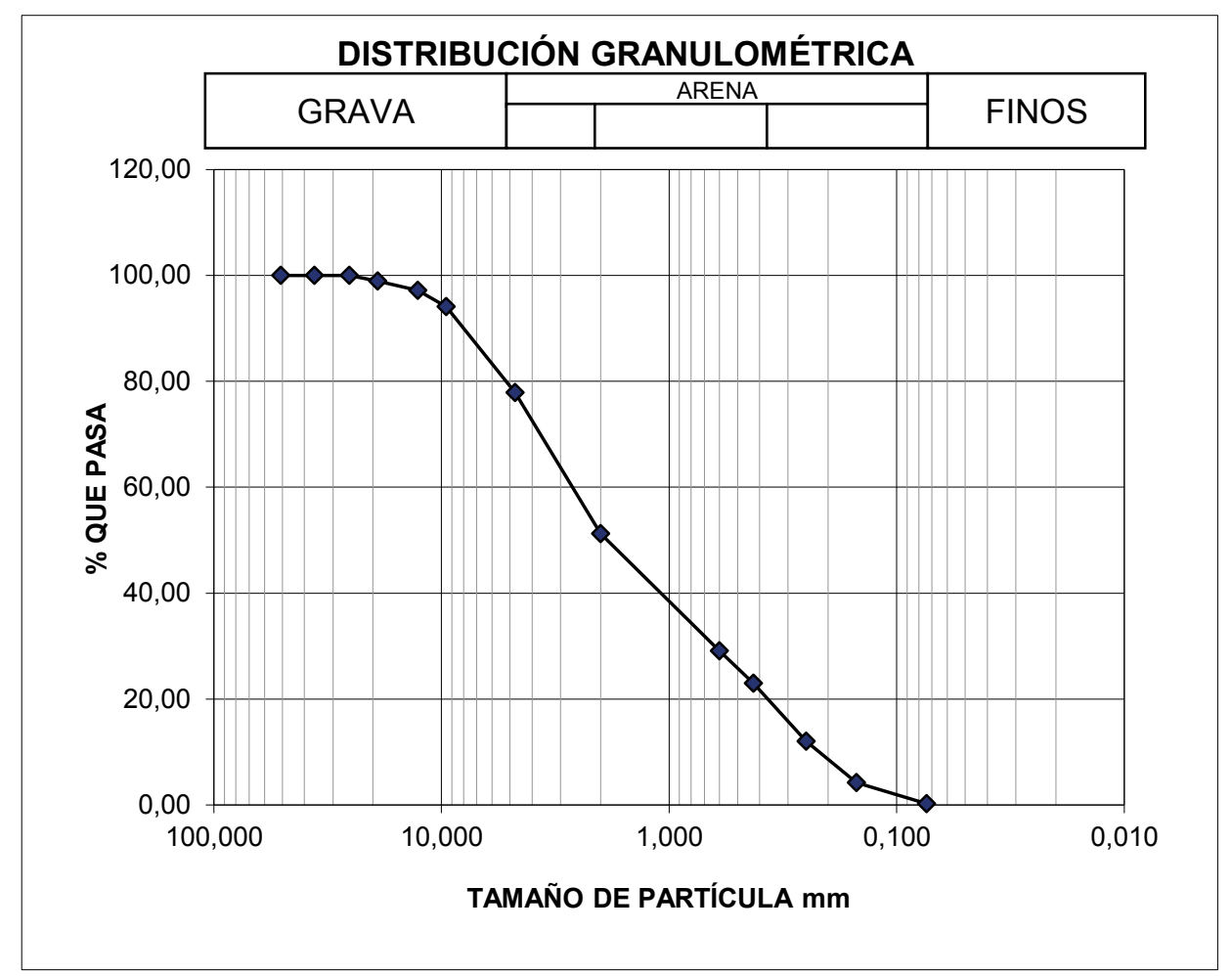

Figura 1. Curva granulométrica agregados reciclados

Fuente: elaboración propia

Tabla 5. Análisis granulométrico agregados naturales

\begin{tabular}{cccccc}
\hline Tamiz N $^{\circ}$ & Diámetro $(\mathbf{m m})$ & Peso Retenido (g) & \% Retenido & \% Retenido acumulado & \% Pasa \\
\hline $2^{\prime \prime}$ & 50,800 & 0,00 & 0,00 & 0,00 & 100,00 \\
\hline $11 / 2^{\prime \prime}$ & 36,100 & 0,00 & 0,00 & 0,00 & 100,00 \\
\hline $1^{\prime \prime}$ & 25,400 & 0,00 & 0,00 & 0,00 & 100,00 \\
\hline $3 / 4^{\prime \prime}$ & 19,050 & 0,00 & 0,00 & 0,00 & 100,00 \\
\hline $1 / 2^{\prime \prime}$ & 12,700 & 1,86 & 1,18 & 0,18 & 99,82 \\
\hline $3 / 8^{\prime \prime}$ & 9,520 & 4,63 & 0,44 & 0,62 & 99,38 \\
\hline 4 & 4,750 & 39,88 & 3,83 & 4,46 & 95,54 \\
\hline 10 & 2,000 & 158,78 & 15,26 & 19,71 & 80,29 \\
\hline 30 & 0,600 & 492,14 & 47,29 & 67,01 & 32,99 \\
\hline 40 & 0,425 & 141,95 & 13,64 & 80,65 & 19,35 \\
\hline 60 & 0,250 & 133,14 & 12,79 & 93,44 & 6,56 \\
\hline 100 & 0,150 & 47,34 & 4,55 & 97,99 & 2,01 \\
\hline 200 & 0,074 & 19,37 & 1,86 & 99,85 & 0,15 \\
\hline Fondo & & 1,18 & 0,11 & 99,97 & 0,03 \\
\hline Total error (\%) & 0,035 & 1040,27 & 99,97 & - & - \\
\hline
\end{tabular}

Fuente: elaboración propia 


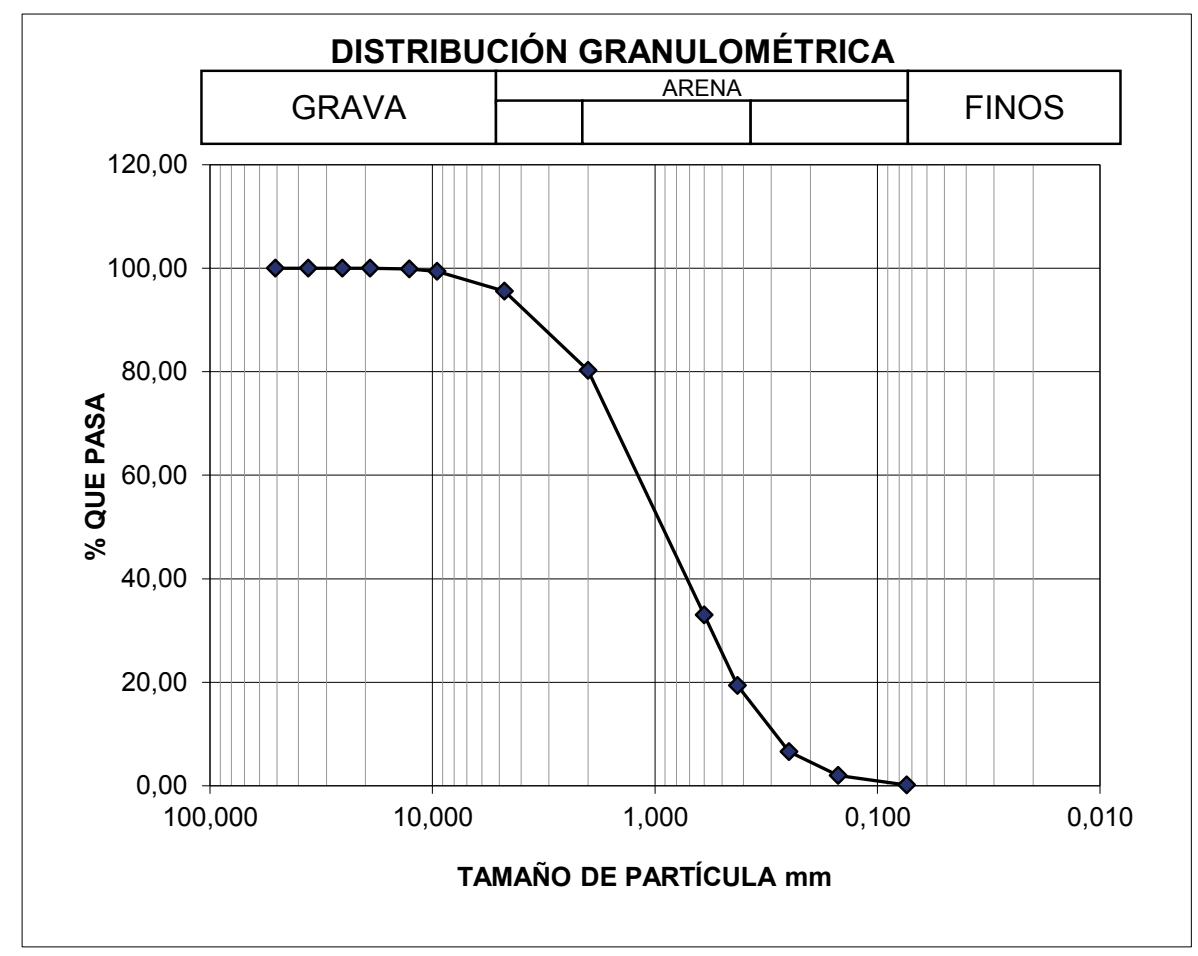

Figura 2. Curva granulométrica agregados naturales

Fuente: elaboración propia

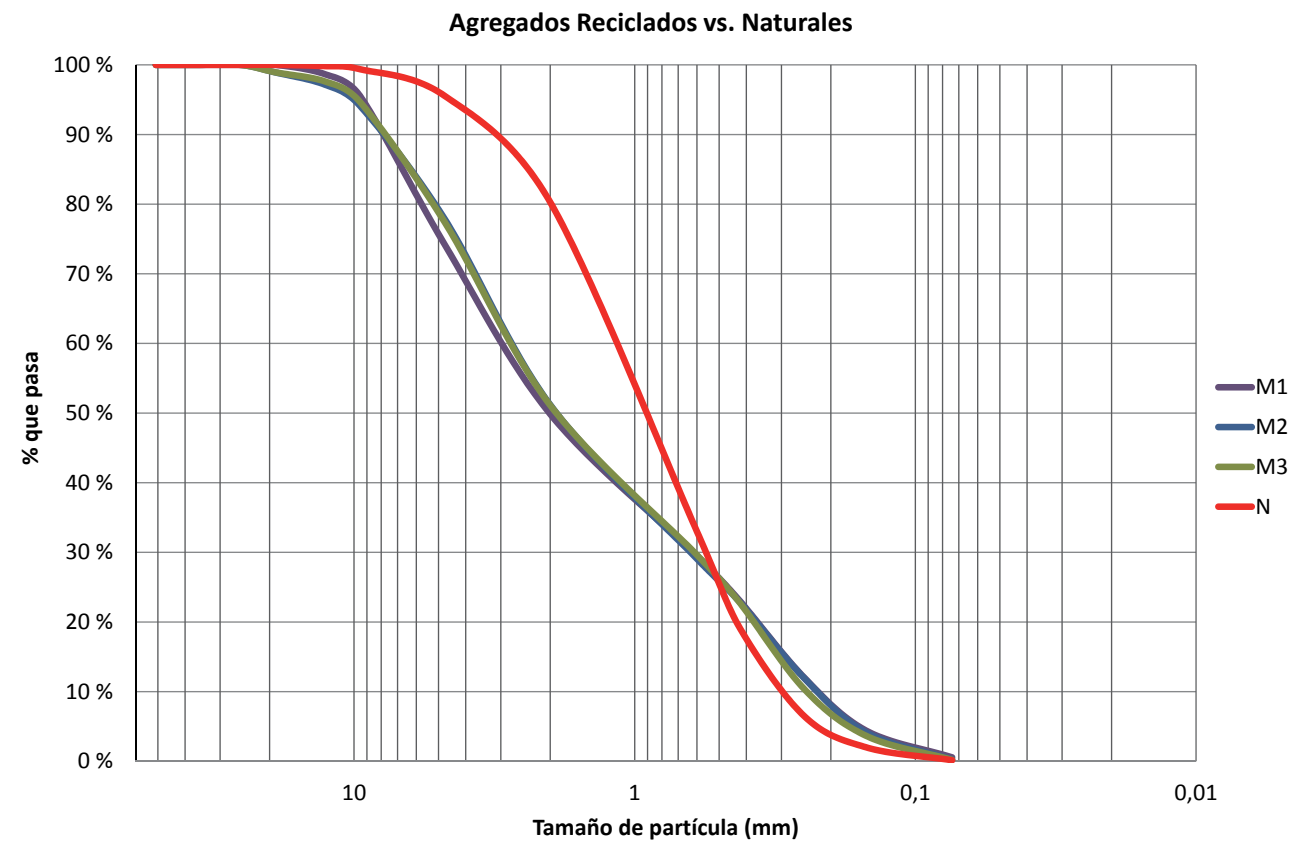

Figura 3. Curva granulométrica de los agregados reciclados respecto a los naturales

Fuente: elaboración propia 
Dado lo anterior, las partículas del agregado reciclado presentaron un tamaño igual o inferior a 3/4" o 19,05 mm; el agregado natural, por su parte, mostró un tamaño aproximado de $1 / 2$ " o $12,7 \mathrm{~mm}$.

En la figura 3, se observa el comportamiento de la curva granulométrica de tres muestras de agregados reciclados $-\mathrm{M} 1, \mathrm{M} 2$ y $\mathrm{M} 3$, respecto a los agregados naturales $\mathrm{N}$-, se pudo observar, que la cantidad de partículas superiores a 0,5 mm, es superior en los agregados naturales. Comportamiento contrario a la cantidad de partículas que se encuentran por debajo de este tamaño, las cuales son superiores en los agregados reciclados.
De acuerdo a los datos presentados en la tabla 2 y la tabla 3, se establecieron los límites a considerar, de acuerdo a los tamices empleados, evidenciando el comportamiento que presentó el agregado reciclado, específicamente la tercera muestra en la figura 4; de esta manera, se establece que la cantidad de partículas inferiores o iguales a 0,6 mm, se ajusta a la normativa. Sin embargo, la cantidad para aquellas que son superiores, es menor al límite inferior establecido.

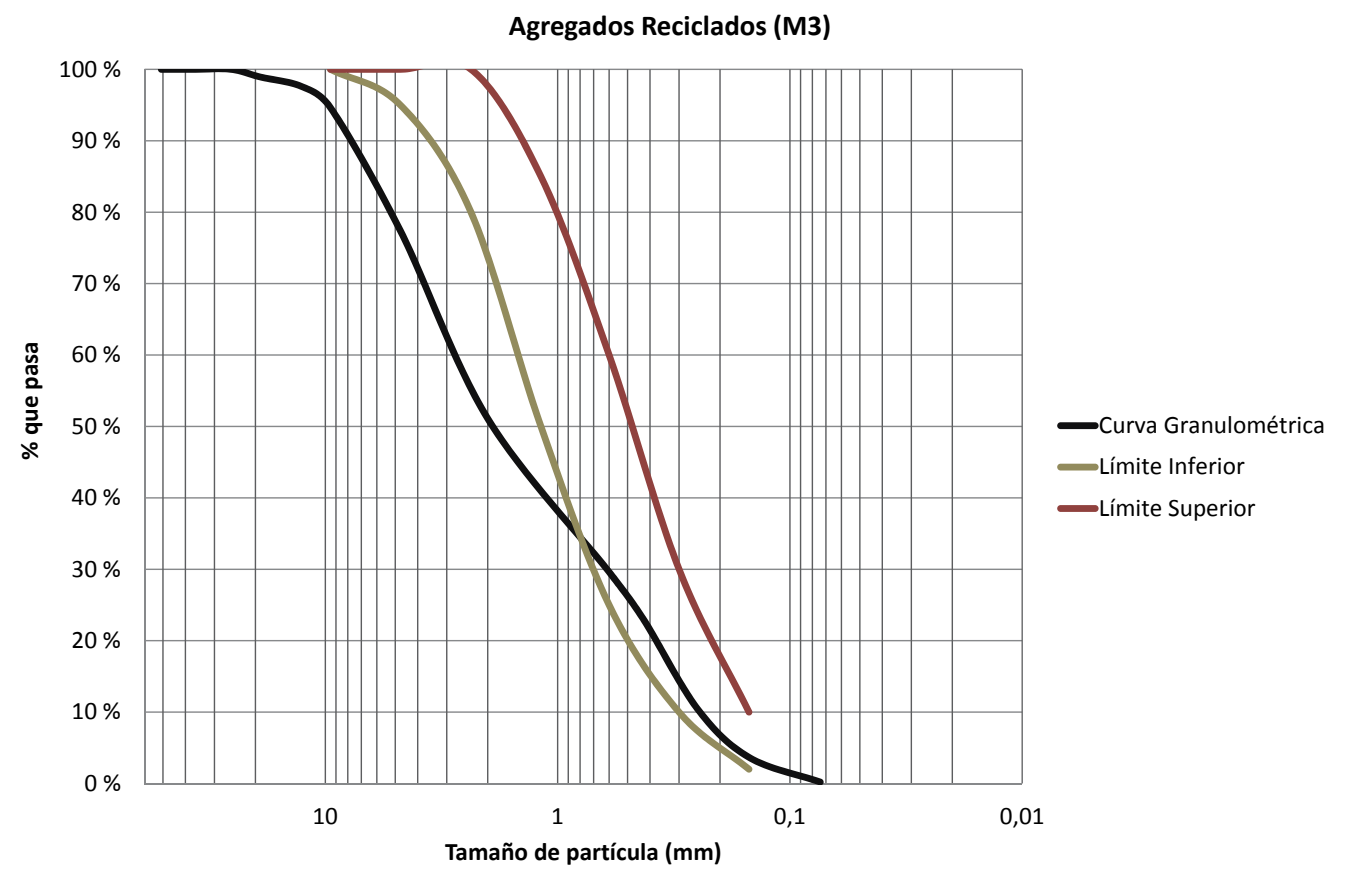

Figura 4. Curva granulométrica de los agregados reciclados respecto a los límites establecidos por la NTC 176

Fuente: elaboración propia

\section{CONCLUSIONES}

De acuerdo a los resultados obtenidos, se verificó que los agregados reciclados no cumplen con las especificaciones dadas por la NTC 174 para los agregados naturales; sin embargo, no debe desestimarse su posible uso como materia prima en la elaboración de elementos para obras civiles, siendo consumidos en proporciones o mezclas apropiadas para cada elemento de mampostería.

Cabe considerar que en esta misma normativa, en el literal 7.2.3, se establece que un agregado 
fino, a pesar de no cumplir con las condiciones dadas en el ensayo, puede ser empleado, si de acuerdo a la NTC 579 "Ingeniería civil y arquitectura. Método para determinar el efecto de las impurezas orgánicas en los agregados finos sobre la resistencia del mortero", la resistencia relativa no es menor del $95 \%$.

En consecuencia, se plantea la posibilidad de realizar en futuras investigaciones, ensayos sobre elementos de construcción elaborados con estos RCD evaluados, con el propósito de validar si su resistencia relativa se ajusta a los límites establecidos por la normativa.

\section{REFERENCIAS}

Banias, G., Achillas, C., Vlachokostas, C., Moussiopoulos, N., and Papaioannou , I. (2011). A web-based Decision Support System for the Optimal Management of Construction and Demolition Waste. Waste Management, 31(12), 2497-2502.

Carrasco, M. F., et al. (2014). Comportamiento higrotérmico de mampostería elaborada conresiduos del desmote del algodón comparación con estructuras tradicionales. III Jornadas de Investigación en Ingeniería del NEA y Países Limítrofes: Nuevos escenarios para la ingeniería en el Norte Grande. Universidad Tecnológica Nacional. 36 - 45pp. ISBN: 978-950-42-0157-1.

Castaño, J., Misle, R., Lasso , L., Gómez, A. y Ocampo, M. (2013). Gestión de residuos de construcción y demolición (RCD) en Bogotá: perspectivas y limitantes. Revista Tecnura, 17(38), 121-129.

Cheng , J., and Ma, L. (2013). A BIM-based System for Demolition and Renovation Waste Estimation and Planning. Waste Management, 33(6), 1539-1551.

De Santos, D., Monercillo, B. y García, A. (2011). Gestión de residuos en las obras de construcción y demolición. Segunda edicion. Madrid: Tornapunta Ediciones. 10-39pp., ISBN: 978-84-15205-28-9.

Ding , T. and Xiao, J. (2014). Estimation of Building-Related Construction and Demolition Waste in Shanghai. Waste Management, 34(11), 2327-2334.
Giraldo, O. (2003). Manual de agregados para el hormigón. Segunda edicion. Medellín: Universidad Nacional de Colombia. Recuperado el 1 de mayo de 2017 del sitio web: http://www.unalmed.edu. co/ ogiraldo/archivos/laboratorio/agregados.pdf

Instituto Colombiano de Normas Técnicas y Certificación (ICONTEC). (2000). NTC 174-Concretos. Especificaciones de los agregados para concreto. Bogotá D.C.: ICONTEC.

Jaramillo, J. (2014). Estudio experimental del comportamiento magnético de multicapas $\mathrm{Cr} / \mathrm{Gd} / \mathrm{Cr}$ no homogéneas. Maestría thesis, Universidad Nacional de Colombia-Sede Manizales.

Kumutha, R., and Vijai, K. (2010). Strength of Concrete Incorporating Aggregates Recycled From Demolition Waste. ARPN Journal of Engineering and Applied Sciences, 5(5), 64-71.

Leiva , C., Solís, J., Marrero , M. and García, C. (2013). Recycled Blocks With Improved Sound And Fire Insulation Containing Construction and Demolition Waste. Waste Management, 33(3), 663-671.

Méndez, N., Rodríguez, C., Cruz, N. y Laines, J. (2010). Los escombros como agregados en la industria de la construcción. Revista de Divulgación de la División Académica de Ciencias Biológicas, 16(30), 37-46.

Méndez, N., Rodríguez, C., Cruz, N. y Laines, J. (2011). Los escombros como agregados en la industria de la construcción. Tabasco, México: Instituto Tecnológico de Villahermosa.

Molina, F., Camacho, J., and Reyes, O. (2016). Stiffness of a Granular Base Under Optimum and Saturated Water Contents. Revista Tecnura, 20(49), 75-85.

Paredes, L., Reyes, O. y Camacho, J. (2014). Comportamiento de mezclas asfálticas densas a partir del ensayo de viga semicircular simplemente apoyada. Revista Tecnura, 18(41), 103-119.

Rivera, G. (2013). Agregados para mortero o concreto. Popayán: Universidad del Cauca.

Sánchez, D. (2001). Tecnología del concreto y del mortero. Bogotá D.C.: Bhandar Editores Ltda.

Scanferla, L. y Barreda, M. (2011). Evaluación del comportamiento de hormigones elaborados con 
agregados reciclados. Buenos Aires: Universidad Tecnológica Nacional.

Srour, I., Chehab, G., El-Fadel, M. and Tamraz, S. (2013). Pilot-Based Assessment of the Economics of Recycling Construction Demolition Waste. Waste Management Research, 31(11), 1170-1179.

Ulsen , C., Kahn , H., Hawlitschek , G., Masini , E., Angulo, S. and John, V. (2013). Production of Recycled Sand from Construction and Demolition Waste. Construction and Building Materials, 40, 1168-1173.

Vega, J. (2013). Sitio de disposición final de Residuos de Construcción y Demolición (RCD). Bogotá D.C.: CEMEX.
Viudaud, I., Castaño , T. y Vidaud, E. (2013). Concreto sustentable ¿mito o realidad? (parte 2). Construcción y tecnología en concreto, 3(5), 20-23.

Wang, C., Wu, J. and Zhang, F. (2013). Development of porous ceramsite from construction and demolition waste. Environmental Technology, 34(15), 2241-2249.

Wu, Z., Yu, A., Shen, L. and Liu , G. (2014). Quantifying Construction and Demolition Waste: An Analytical Review. Waste Management, 34(9), 1683-1692.

Yuan , F., Shen, L.-y.and Li , Q. (2011). Emergy Analysis of the Recycling Options For Construction and Demolition Waste. Waste Management, 31(12), 2503-2511. 